\section{Ueber Geburtshülfe und Gynäkologie in England.}

Von Dr. Paul Strassmann,

Assistenzarzt an der geburtshulflich-gynäkologischen Poliklinik der Kơniglichen Charité zu Berlin.

(Schluss aus No. 46.)

In der Anstellung der Aerzte zeigt sich auch an den gynäkologischen Anstalten das Bestreben, möglichst mehreren eine Hospitalthätigkeit zu gewähren. Am Samaritan Hospital sind vier Surgeons und vier Physicians für die klinischen Patientinnen angestellt. In die Poliklinik theilen sich je drei derselben an je zwei Wochentagen. Die poliklinische Patientin hat ihren Zettel, auf dem oben der Name des sie behandelnden Arztes steht. Mit der Theilung in Surgeons und Physicians geht die Specialisirung auf gynäkologischem Gebiete hier noch einen Schritt weiter. Ein gynäkologischer Surgeon macht nur Laparotomicen, der Physician ist extraperitoneal und behandelt allgemein und operirt per vaginam. Diese Grenze wird ziemlich streng eingehalten, und wenn z. B. eine Laparotomirte abortirt, so übernimmt der Physician die Behandlung des Abortes. Aus der Vertheilung des Materiales an mehrere gleichgestellte Aerzte ergiebt sich ferner, dass nach ganz verschiedenen Principien an demselben Hospital operirt wird. Um nur eins hervorzuheben, so hielt der jüngste Chirurg am Samaritan Hospital, Malcolm, ein Schüler Thornton's, sich noch an alle Vorschriften der Antispsis und operirte z. B. noch unter Carbolspray. Doran, bekannt durch seine mikroskopischen und pathologischen Untersuchungen, und Meredith, zwei an demselben Institut angestellte Operateure, verwendeten Antiseptica etwa in derselben Weise, wie es bei den in Deutschland noch nicht aseptisch operirenden Gynäkologen geschieht, Bantock endlich hat alle antiseptischen Maassregeln wieder aufgegeben und operirt nur nach Reinlichkeitsprincipien, also keineswegs etwa strenger Asepsis. Bantock sah ich etwa 20 Laparotomieen ausführen, darunter einzelne recht unangnehme, grössere, verwachsene Myome, rupturirende Tubensäcke entfernen. Dieselben genasen sämmtlich. Uebrigens war unter etwa $50-60$ nach den verschiedensten Methoden der Asepsis und Antisepsis während meiner Anwesenheit in London am Samaritan Hospital vollzogenen Laparotomieen kein Todesfall. Bantock, der seine Anschauungen in verschiedenen Veröffentlichungen niedergelegt hat, hat recht bemerkenswerthe Resultate erzielt, z. B. eine Serie von 90 Laparotomieen ohne Todesfall.

Ohne Antiseptica, nur nach dem Princip der Reinlichkeit; operirt ausser Bantock noch Laws on Tait in Birmingham, wohl einer der beschäftigtsten Laparotomisten Englands. Bei beiden kommen Antiseptica in keiner Form mehr zur Verwendung, weder am Operateur und der Assistenz, noch bei der Patientin oder an Iıstrumenten und Verbandstoffen. Zur Reinigung der Hände nimmt Bantock nur Wasser, Seife und Bürste, Tait reibt sich vor diesem noch die Hände mit Terpentin gründlich ab. In der gleichen Weise reinigen sich bei Tait die Wärterinnen, bevor sie irgend etwas zur Operation gehöriges anfassen. Die Patientin badet vor der Operation, die Pubes werden nur bei voraussichtlich längerer Incision bis zum Rand der Symphyse abgenommen. Die Bauchdecken werden nicht desinficirt, höchstens mit einem mit Warmwasser getränkten Wattebausch abgerieben.

Während der Operation wird nur erwärmtes, aber nicht gekochtes Wasser verwendet. In diesem werden die Schwämme gereinigt und mit demselben auch die Ausspülung der Bauchhöhle überall da, wo der Austritt irgend welcher Substanzen stattgefunden hat, vorgenommen. Mit Eiter verunreinigte Schwämme werden weggeworfen, die übrigen werden für die nächste Operation durch längeres Spülen und Ausdrücken in heissem Wasser gereinigt. So werden auch die Instrumente behandelt, nach Reinigung in heissem Wasser werden sie gründlich abgerieben und trocken weggelegt, worauf sie ohne Vorbereitung zur nächsten Operation verwendet werden.

Das Catgut wird mit Chromsäure präparirt, die Seide gebrauchte Bantock ohne jede Vorbereitung. Die zu einer Laparotomie nothwendigen Herrichtungen sind dabei freilich schnell getroffen. Es dürfte vielleicht von Interesse sein, einer, die ich bei L. Tait sah, in ihrem Verlaufe zu folgen.

Das Telegramm mit dem Consens des Ehemannes zur Operation traf um $1 / 21$ Uhr Mittags in Birmingham ein, die schriftliche Einladung zur Operation bekam ich eine Viertelstunde später. Gegen $1 \mathrm{Uhr}$ fand ich mich in Tait's Klinik ein. Nach einer weiteren Viertelstunde, während die Patientin narkotisirt und alle Vorbereitungen beendigt waren, begann die Operation. Mit einem dem gewöhnlichen Holzkasten entnommenen Messer, das eine höchstens $1 \frac{1}{2} \mathrm{~cm}$ lange Schneide hatte, wurde ein $4 \mathrm{~cm}$ langer Schnitt in der Linea alba gemacht, die Bauchdecken durchtrennt, das Peritoneum zwischen zwei Péan'schen Klemmchen angehoben, aufgeschlitzt, und der Schlitz mit dem Finger bis zum oberen und unteren Wundwinkel gedehnt. Es handelte sich um frische doppelseitige gonorrhoische Erkrankung der Tuben und Ovarien. Der Operateur ging nun mit zwei Fingern der linken Hand ein und löste die Adhäsionen; da dies auf der einen Seite Schwierigkeiten machte, ging er mit zwei Fingern der rechten Hand in die Scheide und zog nach kurzem combinirten Ausschälen die Adnexe zur Wunde heraus. Die Hand wurde mit Wasser und Seife wieder gereinigt. Das Ligamentum latum wurde jederseits mit nur einem mittelstarken Seidenfaden, der mit stumpfer Nadel durchgeführt wurde, durchstochen und nach Art des Staffordshire-Knotens abgebunden. Nachdem die Adnexe abgetragen waren, wurde der Stumpf, der nicht weiter blutete, mit einem kleinen Schwamm abgewischt und versenkt. Das Peritoneum, an dem sich noch die beiden Péans befanden, wurde angehoben, und die Naht der Bauchwunde geschah nun mit einer geraden Stopfnadel ohne Nadelhalter, mit nur einem Seidenfaden. Der Faden wurde fortlaufend schusternahtartig durch die ganze Bauchwand gelegt, aber nicht angezogen. Das unterste lose Ende wurde zunächst mit dem fortlaufenden lockeren Faden geknotet, der Faden abgeschnitten und das freigewordene Ende in gleicher Weise mit dem fortlaufenden Faden geknüpft, wieder abgeschnitten, und dann der dritte und letzte. Knoten gemacht. Die auf diese Weise durch drei Seidensuturen vereinigte Bauchwunde war kaum so gross, als eine deutsche Probeincision. Etwas gepulverte Borsäure deckte das Ganze, dann kam ein Streifen Gaze und drei Heftpflasterbänder Die Operation hatte, ohne im geringsten den Eindruck der Hast zu machen, acht Minuten in Anspruch genommen. Ein Anaesthetist, ein Assistent und zwei Wärterinnen leisteten die nöthige Beihülfe. Das verwendete Wasser wurde im Kaminfeuer, das gleichzeitig die Stube heizte, erwärmt. Die Patientin beobachtete ich noch fünf Tage, in denen keine Störung oder Temperaturerhöhung eintrat, während weder Alkohol noch Narcotica zur Verwendung kamen.

Nicht nur bei diesen, die Nothwendigkeit antiseptischer und streng aseptischer Maassregeln leugnenden Klinikern, sondern auch bei den meisten sich zur Antiseptik bekennenden Operateuren Englands, und zwar sowohl auf gynäkologisch-geburtshülflichem, wie auf chirurgischem Gebiete, wird sich dem Deutschen sehr bald die Beobachtung aufdrängen, dass keineswegs die strenge $\mathrm{Be}$ folgung aller der von uns für bewiesen angenommenen Grundsätze herrscht. Ist man auch bei uns bereits meistens davon zurückgekommen, die Zuschauer in Weissleinen zu stecken oder sie vorher desinficiren zu lassen, so wird doch noch durchgehends gefordert dass alle Gegenstände, die irgendwie mit den operirenden oder assistirenden Händen in Berührung treten, wie diese selbst vorher besonders einem desinficirenden oder sterilisirenden Verfahren unterworfen wurden. Dies ist in England bei weitem nicht so strict durchgefithrt, und recht oft wird dieser oder jener Gegenstand in dem Bewusstsein, dass er mit niclits Infectiösem direct in Berührung war und daher auch nicht inficiren werde, während der Operation angefasst. Infectionen im bacteriologischen Sinne müssen dabei vorkommen, und trotzdem sind die Laparotomieresultate, wie oben bereits die Wochenbettsstatistiken, recht günstige, wie man sich nicht nur aus den Veröffentlichungen, sondern auch durch persönliche Durchsicht der Bücher und den Augenschein überzeugen kann.

Zwei Factoren müssen hier vor allem ins Gewicht fallen. Erstens müssen wenig Infectionsquellen vorhanden sein, zweitens muss bei möglicher oder vorhandener Infection den Keimen ein thunliehst ungeeigneter Nährboden und nur geringe Gelegenheit, auf diesen zu gelangen, gewährt sein. Die erste Bedingung findet ihre Unterstützung in der bedeutend ausgebildeten englischen Reinlichkeit. Die Einrichtung und Benutzung der Badestuben ist entschieden auch im Volksleben eine weit ausgedehntere, als zur Zeit in Deutschland. Der Gebrauch von Seife spielt in der Hygiene der Briten eine hervorragende Rolle. Die körperliche Reinlichkeit, der wir einen Einfluss auf die Gesundheit der weiblichen Generationsorgane auch bei menstrualen und puerperalen Vorgängen einräumen müssen, spricht sich noch in manchen kleinen Dingen aus. So ist es z. B. allgemein auch bei dem die Polikliniken frequentirenden Publikum eingebürgert, dass die menstruirende Frau ein Tuch (Diaper) sich vorbindet, keinen Diana- oder Moosverband, sondern frischgewaschenes Leinen. Die Quantität des menstrualen Verlustes wird nach der Zahl der gebrauchten Tücher geschätzt. Im St. Bartholomew's Hospital in London hat Dr. Champneys seinen Studenten ein Frageschema für die Anamnese gegeben, worunter die Frage nach der Zahl der Tücher bei den Menses als etwas selbstverständliches aufgezeichnet steht, und Lawson Tait schreibt in seinem Lehrbuche, dass der Gebrauch von weniger als drei und mehr als vier oder fünf innerhalb 24 Stunden oder weniger als zehn und mehr als 15 während der ganzen Zeit einer Menstruation auf Abnormitäten hinweise. - 
Zur persönlichen Asepsis des Operateurs und des Assistenten trägt sicherlich auch mit bei, dass die den einzelnen zur Verfügung stehenden Abtheilungen neist kleine sind, und daher eine Häufungvon mehreren Operationen nicht vorkommt, und dass endlich viele sich so specialisirt haben, dass sie nicht nur die Geburtshülfe, sondern auch alle gynäkologischen Eingriffe, mit Ausnalıme der Laparotomie, aufgegeben haben.

Der zweite Punkt, der Infection einen möglichst geringen und ungeeigneten Nährboden zll gewähren, wird durch verschiedene Maassnahmen unterstiitzt. Der Schnitt wird durchweg möglichst klein gemacht. Man bekommt gar nicht selten Narben von nur $5-6 \mathrm{~cm}$ Länge $\mathrm{zu}$ sehen. Von der durchweg ausgeführten, bei uns von manchen verpönten Punction der Cystome mit dem Troicart habe ich nie Nachtheile erblicken können. Hakenpincetten werden fast nie verwendet, dafür werden kleine Péan'sche Klemmen genommen. Antiseptica werden als solche ïberhaupt nicht in das Cavum abdominis gebracht. Mit Ligaturen wird äusserst sparsam umgegangen. Für die Entfernung der Eierstöcke, gestielter Tumoren, selbst der Tubensäcke wird fast nie mehr als ein Faden verwendet. Der Staffordshire-Knoten ${ }^{1}$ ), der den Stiel oder das ganze Ligament umgreift, sichert die Schlinge vor dem Abgleiten und ist eine ausserordentlich empfehlenswerthe Vereinfachung. Sehr häufig kommt die Drainage zur Verwendung. Mit einem grossen Blechrohr wirl das Abdomen mit warmem Wasser ausgespült, um Blutgerinnsel, Gescliwulstreste oder -Inhalt zu entfernen; besonders die ohne Antiseptica Operirenden wenden diese Ausspülung liäıfig an. Hinterher wird ein Glasrohr eingelegt, und durch dieses werden vor Schluss der Bauchwunde mittels einer mit einem Nélaton'selien Katlieter armirten Spritze etwaige Reste von Spülflüssigkeit aufgesaugt. Tait benutzte hierzu einen Gummiballon mit einem Glasrohr, dessen Mitte sich kugelig ausbaucht, so dass die aufgesogene Flüssigkeit in diesem Behälter bleibt, ohne den Ballon zu verunreinigen und ohne die Aspiration zu unterbrechen. Diese „Drainage tube“ wird frühestens nach zwölf Stunden entfernt, bei blutiger Secretion erst, wenn statt dieser seröse auftritt. Wo Nachblutung gefürchtet wird, ist diese Glastube sehr angebracht, da sie dieselbe sofort nach aussen anzeigt. Um den Verband vor Durchtränkung zu schützen, wird das Ende der Glasröhre durch ein Stück Gummi oder Protectiv gesteckt, ein Schwamm auf die Oeffnung gelegt, und der wasserdichte Stoff darüber zusammengefaltet. Viefach sieht man auch noch, um Durchnässung der Patientin zu vermeiden, bei Ovariotomieen das Gummituch, das durch Heftpflaster auf dem Abdomen befestigt wird und nur ein Oval in der Mitte für die Operation freilässt.

Im grossen und ganzen wird viel laparotomirt. Die Indicationen fïr die Entfernung entzündlich erkrankter Anhänge sind wie anch in Deutschland von den einzelnen Operateuren verschieden weit begrenzt. Die Extrauterinschwangerschaft wird meist operativ in Angriff genommen. Bei Myomen wird von vielen exspectativ verfahren (siehe Thornton's Aufsatz' ${ }^{2}$ ), als Operation wird die Castration bevorzugt, aber auch die Enucleation und supravaginale Amputation vorgenommen. Der Stumpf wird beinahe ausschliesslich extraperitoneal versorgt. Die Constriction wird mittels Draht häufig gemacht, es wird dazu eine Deltametall genannte Legirung verwendet, als Schnürer dient der Serre noeud von Koeberlé. Eine Vernähung des Stumpfes und Fixirung desselben mittels Naht wird nur ausnahmsweise vorgenommen. Gewöhnlich werden armirte Spicknadeln zum Hochhalten durchgestossen. Der constringirende Draht bleibt liegen und fällt später ab.

Was die Nachbehandlung bei Laparotomirten betrifft, so kommen Alkoholica und Narcotica sehr wenig zur Anwendung. Die letzteren vornehmlich bei Myomotomieen, bei denen die Constriction liegen bleibt. Bei den ersten Symptomen eintretender Peritonitis will Lawson Tait von der Darreichung starker Abführmittel gute Erfolge gesehen haben.

Statistisch sei hier angeführt; dass von 35 Myomoperationen (1890) am Samaritan Hospital 11 Castrationen waren mit drei Todesfällen, 24 supravaginale, extraperitoneale Amputationen ohne Todesfall.

Ueber eine sehr grosse Zahl von Bauchschnitten verfügt die Frauenklinik in Birmingham, an der ausser Tait und seinem Assistenten Dr. Martin noch Dr. Savage und Dr. Taylor operiren. Nach dem Jahresbericht $1890 / 91$ fanden von 1884 bis 1890912 Laparotomieen mit 56 Todesfällen $=6,14 \%$ statt. Unter diesen befanden sich

Todesfälle

8 Hepatotomieen

21 Nierenoperationen $(\dot{6}$ Nephrectomieen $)$

31 Exstirpationen ectopischer Schwangerschaft

1

No. 31

1) S. Spencer Wells, Volkmann's Sammlung klin. Vorträge, N. F.

2) Brit. med. Journ. 11. Februar 1893.
183 wegen Ovarialcystom

22 wegen Parovarialcysten

8.5 wegen doppelter Pyosalpinx

8 wegen einseitiger Pyosalpinx

124 wegen "chronischer Ovaritis"

endlich 92 Castrationen wegen Myomen

dagegen 16 Hysterectomieen wegen Myomen

So hoch die Banchchirurgie in England und Schottland ausgebildet ist, so sehr tritt die operative Scheidengynäkologie in den Hintergrund. Schon dass an den grossen Specialanstalten eine Scheidung nach Surgeons und Physicians möglich ist, weist darauf hin, dass per abdomen mehr operirt wird als per vaginam. An den Medicinschulen ist natürlich eine solche Tremnung nicht durchgeführt. Ein sehr grosser Werth wird auf die Allanınese und die äussere Untersuchung gelegt. An die in England übliche gynäkologische Untersuchung hat man sich als Deutsclier erst sehr zu gewöhnen. Die Mehrzalıl untersucht in Seitenlage, gar nicht selten hat man sich noch unter der Decke bei nur zurückgeschlagenen Kleidern durch die Wäsche hindurchzuarbeiten. Eine combinirte Untersuchung ist in dieser Lage sehr schwer durchführbar. Viele lassen nach der Untersuchung in Seitenlage die Franen sich in Rückenlage drelien und untersuchen dann auf dem Tisch oder je naclidem im Bette so, dass die Hand bei aufgestellten, geschlossenen Schenkeln eindringt. Der Untersuchungsstuhl beginnt sich langsam einzubürgern, er war unter anderem im Samaritan Hospital, im St. Thomas Hospital und an den Dubliner Kliniken in Gebrancli. Viele Aerzte sagten mir, dass man sich mit dieser Lage selir bald die englischen Frauen alls der Sprechstunde jagen würde.

Man kann recht lange in England sein, ohne eine grössere Operation von der Scheide aus zu sehen zir bekommen. Die Exstirpation des malign erkrankten Uterus auf diesem Wege war bei der grösseren Zalil der Operationen eine rara avis, deren während dreimonatlichen Aufenthaltes in London ansichtig zu werden, mir nicht gelang. Grössere Reihen dieser Operation sind, soweit mir bekannt, niclit viel veröffentlicht ${ }^{1}$ ). In Edinburgh referirte Dr. Brewis über zwei von ihm wegen Sarkom per vaginam exstirpirte Uteri. Bei Krebs des unteren Gebärmutterabschnittes bevorzugt man die hohe Amputation.

Ueberraschend für den Deutschen ist es, dass der Prolaps in England so wenig operativ behandelt wird. Frische und für operative Behandlung ausserordentlich geeignete Prolapse, bei denen die Patientinnen selbst um Operation baten, sah icl gar nicht selten abgewiesen werden und mit den verschiedensten Formen von Pessarien oder Hysterophoren weggeschickt. Eins der Hysterophore, das Napier'sche, schien mir, wo überliaupt ein derartiges Instrument $\mathrm{zu}$ verwenden ist, recht praktisch. Sehr viele Operateure führen die Colporrhaphie wirklich nur bei alten Prolapsen aus. Man scheint sicl hier weniger um die geringen Gefahren als über den Endeffect zu besorgen. Eines Falles erinnere ich mich, wo bei Prolapsus vaginae et uteri nach vergeblicher Anwendung von Pessarien bereits die Frage der Ventrofixation ven tilirt wurde, nur um die Colporrhaphie bei einer Patientin il jüngerem Alter zu vermeiden. Kein Wunder, wenn dann der Edinburgher Anatom Dr. Sy mington besonders schöne Gefrierschnitte bei completem Prolaps demonstriren kann! In der Behandlung des Prolapses werden sich wohl die britischen Anschallungen noch ändern.

L. Tait verwendet Pessare übrigens gar nicht mehr bei Prolaps, er hat alle Colporrhaphieen aufgegeben und macht nur seine Lappendammplastik mit dem bekannten H-Schnitt. Für den alleinigen Descensus der vorderen Vaginalwand macht er neben den begünstigenden Momenten des Wochenbettes etc, vor allem die habituelle Ueberdehnung der Blase bei der Frau verantwortlich, und die Behandlung bestelit daher in der Vorschrift der häufigen Entleerung der Blase.

Die elektrische Behandlung der Frauenleiden ist von vielen, einer Debatte in der Obstetrical Society nach zu schliessen, nocl gar nicht versucht worden. Bekannt dürfte sein, dass Keith, der die grösste Anzahl von Hysterectomieen bei Myomen ausgeführt hat (mit einer Mortalität von nur $4 \%$ ) das Messer gänzlich mit der Elektrode vertauscht hat und gute Resultate dabei haben will. Gerade im Frühling 1892 machte sich eine lebhafte Strömung gegen die Apostoli'sche Behandlung geltend.

Die Massage nach Thure Brandt wurde in England niclit geübt und bedauerlicherweise oft als Masturbation verworfen.

Ueberhaupt wird vaginal mit Tampons, Pinselungen, Scarificationen in den Polikliniken sehr wenig behandelt. Es hiess die Frauen würden sich das nicht lange gefallen lassen. Hier wird medicamentös und exspectativ verfahren. Ich stehe nicht an zu bemerken, dass dabei Menstruationsanomalieen, Perimetritiden, metri- 
tische Erscheinnungen und Lenkorrhoeen der verschiedensten Art anch ohne locale Behandlıng sich besserten, und manchunal stieg die Empfindung anf, ob nicht in Deutschland bisweilen in dieser Beziehuilg des Guten zu viel geschehe.

In grossen Zügen habe ich ver'sucht, die fïr den augenblicklichen Standpunkt der Geburtshïlfe und Gynäkologie in England charakteristischen Merkniale wiederzugeben nlld von den wichtigsten, sich von dentschem Branche scheidenden Einzelheiten ein Bild zn entwerfen. Gerade sie geben diesen Theile der Medicin noch eine Art nationalen Gepräges und sind in ihrer Entwickelnng, wein man Gelegenheit hatte, Land nnd Leuten näherzııtreterl, wohl verständlich. Dass hierbei dem ärztlichen Besucher alle Wege offell stehen nlld echte Collegialität ihn bei seinen Wanderungen nnd Forschungen fïhrt nnd nniterstiitzt, muss man mit dem aufrichtigsten Danke anerkennen. 